\title{
Using nature-based soundscapes to support task performance and mood
}

\section{Joseph W.Newbold}

University College London

London, UK

joseph.newbold.14@ucl.ac.uk

\section{Jake Luton}

University College London

London, UK

jacob.luton.15@ucl.ac.uk

Anna L. Cox

University College London

London, UK

anna.cox@ucl.ac.uk
Sandy J. J. Gould

School of Computer Science

University of Birmingham

Birmingham, UK,

s.gould@cs.bham.ac.uk

Paste the appropriate copyright/license statement here. ACM now supports three different publication options:

- ACM copyright: ACM holds the copyright on the work. This is the historical approach.

- historical approach. exclusive publication license.

exclusive publication license. access. The additional fee must be paid to ACM.

This text field is large enough to hold the appropriate release statement assuming it is single-spaced in Verdana 7 point font. Please do not change the size of this text box.

Each submission will be assigned a unique DOI string to be included here.

\begin{abstract}
Smartphone apps that enable workers to listen to nature soundscapes are increasingly popular. There is, however, little evidence that these soundscapes have the effects that they claim to have. Previous research exploring the effect of listening to background music during tasks has shown that while such music may have a positive effect on emotional state, it can disrupt reading and memory-based tasks. This paper explores the effects of nature soundscapes on mood and performance. A diary study of the use of soundscapes whilst studying suggests that students view such soundscapes as: aiding focus whilst studying; creating feelings of calm and peace; helping to manage stress and anxiety; and hiding distracting sounds. A second study - an experiment - investigated the effects of nature soundscapes on mood and performance. Whilst we found no effect of soundscapes on mood and arousal during the task, our results demonstrate that high acoustic variation in a soundscape may cause a disruption to serial recall tasks. The implications of our findings suggest that nature soundscapes with high acoustic variation may be detrimental to task performance compared to working in silence for serial based thinking tasks.
\end{abstract}




\section{Author Keywords}

Soundscapes; nature; workplace; task performance; mood;

\section{ACM Classification Keywords}

H.5.m. Information interfaces and presentation (e.g., $\mathrm{HCI}$ ): Miscellaneous;

\section{Introduction}

App stores are full of apps promising to help people focus by playing them ambient sounds (e.g. Nature Sounds [1] has between 500,000-1,000,000 installs). Ambient sounds, which include background music and nature-based soundscapes, aim to limit distraction and increase productivity [2,3]. In addition to simply masking disruptive external noise, music and soundscapes can both also improve mood, relaxation and performance within individual tasks $[4,5]$. However it has also been shown that these sounds may disrupt and lower performance for certain tasks, e.g. serial thinking tasks [6]. Gifford argues that in order to be beneficial, ambient soundscapes need to be matched to specific tasks [7]. Gifford does not describe how this matching should be undertaken or how large we should expect the effects of matching to be. In this paper, we present our initial efforts to investigate the specific effects of different types of soundscape on task performance, mood and arousal. We also explore the broader experience of studying whilst listening to nature-based soundscapes.

\section{Sound Variation}

While background sound might be a largely useful to aid working, one particular aspect of sound has the potential to detrimentally affect people's performance. Due to our ear's constant monitoring of the sounds happening around us, sounds that we pick up over the background noise level can distract us from or current tasks [25]. This means that sounds with a high acoustic variation, such as environmental sounds in an office or music which changes significantly with each beat, are especially distracting $[6,9]$.

\section{Ambient Audio in the Workplace}

Background sound has been shown to mask disruptive environmental sounds [2], aid productivity [3] and improve mood [4]. This may be part of the reason that listening to background music during work tasks has become popular with young people [8]. Moreover, listening to music excerpts before a task has been shown to have positive impacts for both a variety of musical stimuli and for a range of tasks [4]

However, Kämpfe et.al's [9] meta-analysis of the effect of listening to background music during tasks has shown that while it may have a positive effect on emotional state, it also disrupts reading and memorybased tasks. It has also been shown that other sounds with high acoustic variation also disrupt performance in cognitively demanding tasks [10]. As demonstrated by Perham and Vizard, even people's preferred music may be disruptive [6]. This can be explained by the changing state hypothesis: auditory distractors that include a high amount of acoustic variation have a greater interfering effect on the serial recall of information in short-term memory [11] than those with low acoustic variation.

Most of the research that has considered the impact of auditory stimuli in task performance has focused on the effects of listening to music. However, natural soundscapes (i.e. rainfall/birdsong) have become 
"...I prefer some sound around my ear to block the noise of other people in the environment.

$\mathrm{P1}$ - Interview(Q1)

"It made me less stressed, I think. Hearing the birds and the running water in the background triggered me to imagine myself sitting on a river bank in the woods, reading or just relaxing ... so I think these nature sounds did help a bit to calm my nerves."

$$
\text { P2- Diary(Q2) }
$$

"...reminded me of taking nature walks back home; I used to go to the mountains ... This reminder is nice because I really love going to the mountains."

$$
\text { P2 - Diary(Q3) }
$$

"It made me feel distant from the environment I was in ... It also gave me a sense of stability and consistency."

$$
\text { P4 - Diary(Q4) }
$$

increasingly popular in office environments [12] and seem to have relaxing effects [5]. In addition, the more continuous nature of these soundscapes (i.e. the lower acoustic variation of them compared to sounds such as music that have high acoustic variation), may limit the disruption experienced during work tasks [13].

\section{Study 1: Diary Study}

A diary study was conducted to gain a better understanding of how different kinds of tasks might be impacted by the use of nature-based soundscapes. We also wanted to know how people use soundscapes in the wild.

\section{Participants}

Four participants took part in the diary study ( 3 male, 1 female). Participants were paid $£ 25$ (USD 30) for participation. The study required participants to engage in periods of study for at least forty minutes. The periods had to include both reading and writing.

\section{Materials}

Participants created soundscapes using nature sounds in the sound mixer at naturesoundsfor.me [21]. This sound mixer provides 13 continuous background nature sounds (rain, waterfall, etc.) and 19 intermittent sounds (woodpeckers, sheep, etc.). Up to four kinds of sound can be overlaid on each other. The study environment was chosen by participants, but they were asked to include both public and private locations.

\section{Procedure}

Participants in the diary study were asked to use nature soundscapes on at least four days of a 7-day period. On each day, participants were asked to construct their own nature soundscape, using the sound types described above. Participants played the soundscape whilst simultaneously engaged in at least 40 minutes of study activity, which included both reading and writing. The participants spent 20 minutes making a diary entry within one hour of the study period. Afterwards, the participants had an exit interview in which they could explain their diary entries, enabling a deeper understanding of the participants' experience and to clarify any apparent ambiguities. The data were then analysed using a six-stage thematic analysis technique outlined by Braun and Clarke [22]

\section{Results \& Discussion}

Three overarching themes emerged from the thematic analysis: a sense of control over the environment and self, incongruence between sound and place and mental stimulation through nature soundscapes.

\section{Control over self and environment}

In general, participants reported that nature soundscapes had a positive impact on their ability to study. The nature soundscapes were both cognitively stimulating and acted as a sound masker. Most participants reported that soundscapes were useful for achieving feelings of calm and peace. The use of nature soundscapes as a sound masker was also frequently referred to when studying in an open public place (see Q1).

Participants also used nature soundscapes with the intention of controlling or affecting their mood. Overwhelmingly, this was to create feelings of calm and peace and to manage stress and anxiety (see Q2). 
P5: Yeah, it sort of didn't make any sense because, so what I realised is that they, like the, they're very effective in taking you out of context of your physical

environment..."

$\mathrm{P} 4$ - Interview(Q5)

"I prefer working in a place where there is a little bit of ambient noise in the background, and like completely silent places kill me, and so in silent places that's where I usually have headphones on like playing a little bit of something"

P3 - Interview(Q6)
Incongruence between sound and place

The second theme was the effect on participants brought about by the juxtaposition of the nature soundscapes compared to the study environment. This was found to create both positive and negative effects. Positive effects seemed to be based on the soundscapes reminding the participants of times when they were connected to a natural environment (see Q3 and Q4). Negative effects including uneasiness and discomfort were also found when participants considered the effects of the soundscapes (see Q5)

Mental stimulation through nature soundscapes Participants' descriptions of using soundscapes were linked to maintaining focus and attention. All participants reported that they felt that they worked better when they had some background stimulation (see Q6).This matches previous research that showed that auditory stimulation can help to maintain attention $[23,24]$.

Given that the participants in our diary study had a positive experience and felt that the use of nature soundscapes positively impacted both mood and performance whilst studying, we conducted an experimental study to investigate whether such effects could be found through objective measures and specifically to investigate the impact of acoustic variation within nature soundscapes.

\section{Study 2: Laboratory Experiment}

In our second study, we investigated whether naturebased soundscapes with different levels of acoustic variation affect mood, arousal and task performance in a mental arithmetic task. This task was chosen as a task that requires serialisation in working memory; therefore we would expect to see a detrimental impact of high acoustic variation on performance (known as the Irrelevant Sound Effect or ISE). As such the three conditions used were silence (control), birdsong (a naturalistic soundscape with high acoustic variation and rain (a naturalistic soundscape with low acoustic variation). We, therefore, expect that:

- H1: a high level of acoustic variation in the nature soundscape decreases performance in a task that requires serial recall.

- H2: listening to nature soundscapes increases positive mood, cognitive arousal and task performance compared to control) and

\section{Method}

Participants

18 participants (10 male) aged between 23 and 36 years ( $M=28$ years) participated in the study.

Participants were included in a draw to win either a prize of $£ 50$ or one of two of $£ 25$

Design

The experiment employed a within-subject design. The independent variable was environmental sound and had three conditions: silence, birdsong soundscape and rain soundscape. These were counterbalanced across participants. The dependent variables were performance on a mental arithmetic task (number of correct answers given), self-reported mood, and selfreported arousal (both measured using POMS [14]).

\section{Materials}

The arithmetic task was presented on a desktop computer with a 17-inch screen. On the corners of the desk were two Dell AX210CR external speakers. The 


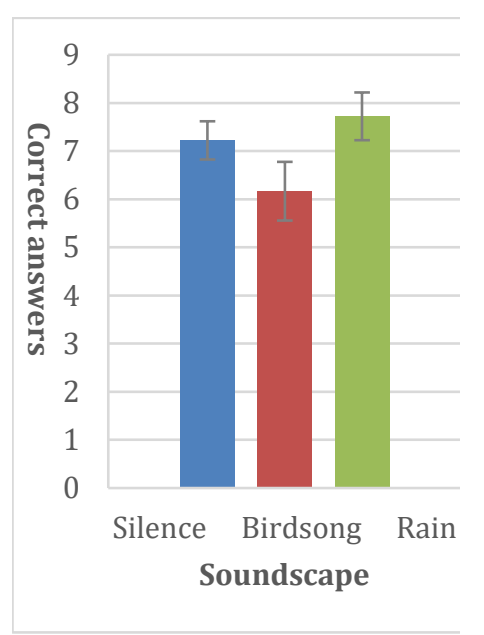

Figure 1: Figure showing task performance results across conditions

\begin{tabular}{l|ll}
\multicolumn{1}{c}{} & \multicolumn{1}{c}{ Mood } & \multicolumn{1}{c}{ Arousal } \\
\hline Silence & $\begin{array}{l}\text { A } 0- \\
\text { 3.25) }\end{array}$ & $\begin{array}{l}5(0.75- \\
10.25)\end{array}$ \\
Bird- & $0(0-1)$ & $7(1-11.25)$ \\
song & 0 & \\
Rain & $0(0-$ & $4.5(3-9.5)$ \\
& $2.25)$ &
\end{tabular}

Table 1: Median (IQR) for POMS measures across the conditions sound volume was configured so that the speakers produced the soundscapes at 60 decibels, approximately the level of a conversation in a restaurant/office [15]. This volume is within the range identified as capable of producing the ISE [16].The soundscapes were produced using audio files from the British Library $[17,18]$. The soundscapes were looped so that they would last for the duration of the experimental session. The low acoustic variation condition was of rainfall. The high acoustic variation condition was of birdsong.

The mental arithmetic task replicated that used by Perham et al. [19], comprising 10 addition runningtotal arithmetic problems. The problems that comprised each of the three tasks were randomised for each participant. The task was created, presented, and completed by the participants using an interactive PowerPoint presentation.

Arousal and mood were measured using the Profile of Mood States (POMS) - Short Form [14]. Although only measures of arousal and mood were analysed in the current study, the entire questionnaire was presented in order to preserve its validity.

\section{Procedure}

The soundscapes, if any, were already playing in the laboratory each time the participant entered for each session. Before the first session, the participants completed one practice trial of the mathematical problem so that they understood the task. At the end of each session, the participant was asked to complete the POMS. The participants took approximately 15 minutes to complete each of the three sessions and received a 5-minute break between conditions.

\section{Results}

An overview of the results can be found in Figure 2 and Table 2.

\section{The impact of acoustic variation on task performance}

A one-way repeated measures ANOVA was conducted to compare the effects of background nature soundscapes on the number of correct answers in a mental arithmetic task. The results show that there was a significant effect of background soundscape on the number of correct answers in the mental arithmetic task $F(2,34)=5.57, p=0.008, \eta_{2}=.247$.

Three posthoc comparisons were made using paired samples t-tests between conditions. A Bonferroni correction was applied. A first paired samples t-test revealed that there was no significant difference between silence and birdsong playing as a background soundscape; $t(17)=2.133, p=0.143$. A second paired samples t-test revealed that there was no significant difference between silence and rain playing as a background soundscape; $t(17)=1.144, p=0.806$. A third paired samples $t$-test revealed that there was a significant difference between birdsong and rain playing as a background soundscape; $t(17)=3.154, p=0.017$. Therefore, these results suggest that birdsong negatively impacted performance in the mental arithmetic task when compared to a rain soundscape. However, no significant difference in performance was found in either birdsong or rain soundscapes compared to silence. 


\section{The impact of nature soundscape on mood and} arousal

The data from the POMS questionnaire were analysed with a Friedman test. For the mood subscale of responses, no significant difference was found between either of the soundscape conditions and silence $X^{2}(2)=$ $1.102, p=0.576$. For the arousal subscale of responses, no significant difference was found between either of the soundscape conditions and silence $x^{2}(2)=$ $0.123, p=0.940$. Whilst the median average subscale measures of arousal suggest a slight trend of birdsong having a positive impact on arousal, this difference was not significant.

\section{Discussion}

While task performance was not increased in comparing either soundscape or silence, it was found that the birdsong-based (high acoustic variation) soundscape did negatively impact the task when compared to the rain soundscape $\mathbf{H 1}$. This matches findings that music, which also has a high acoustic variation, can disrupt serial based thinking [6].

These findings suggest that when thinking about how different background sounds affect various tasks, that soundscapes with limited acoustic variation will be more suitable for serial cognition tasks. As suggested by Gifford [7], evolving soundscapes like these may better support task switching, providing the soundscapes used are appropriate for the task at hand.

These results demonstrate no significant effect of the soundscapes on mood or arousal compared to the silence H2. This contrasts with previous work that demonstrated that the use of natural elements in technology and workplace design may have a beneficial impact on wellbeing [20] which was not seen here, at least in the short term. However, no negative impact was seen from introducing the soundscapes, so they could still prove useful in the masking of outside disturbances [2]. Additionally, as was seen in the diary study, participants did feel an impact on their mood. Therefore it could be the given experiment was unlikely to replicate the in situ experience of working in an office environment where workers may suffer from low motivation, and fatigue.

However, further study is needed to see what other aspects of nature-based soundscape affect different kinds of work tasks in situ.

\section{Conclusion}

This work describes an initial pair of studies that investigate how nature-based soundscapes may be used within a workplace environment to affect mood, arousal and task performance. The diary study demonstrated how nature-based soundscapes may be used in situ and how the people perceive their impact on their work performance and mood. The experimental work then demonstrates how high acoustic variation in this kind of soundscape may cause a disruption to serial recall tasks. Overall this work shows how certain aspects of soundscapes, while they may be viewed positively, can negatively impact performance of certain tasks. Further research is needed to see how different types of soundscapes may be used to support different work tasks. 


\section{References}

1. Nature Sounds on Google Play Retrieved January 8, 2017 from

https://play.google.com/store/apps/details?id=net. metapps. naturesounds\&hl=en

2. Alana G. DeLoach, Jonas Braasch and Jeff $P$. Carter. 2015. Tuning the Cognitive Environment: Sound Masking with 'natural' Sounds in Open-plan Offices. Acoustical Society of America, 137(4).

3. Frances H. Rauscher, Gordon L. Shaw and Catherine N. Ky. 1993. Music and spatial task performance. Nature, 365, 611.

4. E. Glenn Schellenberg, Takayuki Nakata, Patrick G. Hunter and Sachiko Tamoto. 2007. Exposure to Music and Cognitive Performance: Tests of Children and Adults. Psychology of Music, 35(1), 5-19.

5. Helena Jahncke, Staffan Hygge, Niklas Halin, Anne Marie Green and Kenth Dimberg. 2011. Open-plan office noise: Cognitive performance and restoration. Journal of Environmental Psychology, 31(4), 373-382.

6. Nick Perham and Joanne Vizard. 2011. Can preference for background music mediate the irrelevant sound effect? Applied Cognitive Psychology, 25(4), 625-631.

7. Toby Gifford 2016. Tuning into task sonic environmental cues mental task switching. In Proceedings of the 22nd International Conference on Auditory Display (ICAD-2016)

http://www.icad.org/icad2016/proceedings2/paper s/ICAD2016 paper_39.pdf

8. Mark L. Carrier, Nancy A. Cheever, Larry D. Rosen, Sandra Benitez and Jennifer Chang. 2009. Multitasking across generations: Multitasking choices and difficulty ratings in three generations of Americans. Computers in Human Behavior, 25(2), 483-489. http://dx.doi.org/10.1167/10.7.260.

9. Juliane Kämpfe, Peter Sedlmeier, and Frank Renkewitz. 2010. The impact of background music on adult listeners: A meta-analysis. Psychology of Music, 39(4) 424-448

10. Simon Banbury and Dianne Berry. 1998. Disruption of office-related tasks by speech and office noise. British Journal Of Psychology, 89, 499-517.

11. Dylan Jones, Clare Madden and Chris Miles, 1992. Privileged Access by Irrelevant Speech to Shortterm Memory: The Role of Changing State. The Quarterly Journal of Experimental Psychology Section A, 44(4), 645-669.

12. Jesper J. Alvarsson, Stefan Wiens and Mats E. Nilsson. 2010. Stress Recovery during Exposure to Nature Sound and Environmental Noise. International Journal of Environmental Research and Public Health, 7(3), 1036-1046.

13. Phillip C. Beaman, Dylan M. Jones and James $\mathrm{H}$. Neely. 1997. Role of Serial Order in the Irrelevant Speech Effect: Tests of the Changing-State Hypothesis. Journal of Experimental Psychology: Learning, Memory, and Cognition, 23(2), 459-471.

14. Douglas M. McNair, Maurice Lorr and Leo F. Droppleman. 1971. Manual for the Profile of Mood States. San Diego, CA: Educational and Industrial Testing Services.

15. IAC Library. 2017. Comparitive Examples of Noise Levels Retrieved January 10, 2017 from http://www.industrialnoisecontrol.com/comparative -noise-examples.htm

16. Sébastien Tremblay, Dylan M. Jones and Thomas H. Carr. 1999. Change of Intensity Fails to Produce an Irrelevant Sound Effect: Implications for the Representation of Unattended Sound. Journal of Experimental Psychology: Human Perception and Performance, 25(4), 1005-1015.

17. Phil Riddett. 2012. Weather - rainfall. Audio. British Library Sounds. Retrieved 7 August 2016, from http://sounds.bl.uk/Environment/Weather/022MWA12015X0002-0045V0 
18. Kyle Turner. 1989. Downland Scrub. European wildlife 01/56. Audio. British Library Sounds.

Retrieved 7 August 2016, from http://sounds.bl.uk/ Environment/Soundscapes/022M-W1CDR0001064o300V0.

19. Nick Perham, Helen Hodgetts and Simon Banbury. 2013. Mental arithmetic and non-speech office noise: An exploration of interference-by-content. Noise and Health, 15(62), 73-78.

20. Miles Richardson, Marta Maspero, David Golightly, David Sheffield, Vicki Staples and Ryan Lumber. 2016. Nature: A new paradigm for well-being and ergonomics. Ergonomics, 1-14.

21. naturesoundsfor.me Retrieved 10 January 2017, from naturesoundsfor.me

22. Virginia Braun and Victoria Clarke. 2006. Using thematic analysis in psychology. Qualitative Research in Psychology, 3 (2). 77-101. ISSN1478088.

23. Goran Söderlund, Sverker Sikstrom and Andrew Smart. 2007. Listen to the Noise: Noise Is Beneficial for Cognitive Performance in ADHD. Journal of Child Psychology and Psychiatry, 48(8), 840-847.

24. Sverker Sikström, Anna-Maria Jürgensen, Maryam Haghighi, Daniel Månsson, David Smidelik, D and Thomas Habekost. 2016. Self-Rated Attentiveness Interacts with Transcranial Direct Current

Stimulation and Noise Stimulation in Reaction Time in a Go/No-Go Task. 2016, 5

25. Donald J. Tellinghuise and Erin J. Nowak. 2003. The inability to ignore auditory distractors as a function of visual task perceptual load. Perception \& Psychophysics 65(5). 817-828

doi:10.3758/BF03194817 